\title{
Marriage Of Focused and Broad Ion Beam: Sample Preparation Optimized for High Performance Analytical (S)TEM
}

\author{
Max V. Sidorov \\ Materials Technology Development, Advanced Micro Devices, Sunnyvale, CA 94088-3453
}

Since introduction [1], FIB systems are progressively more used for TEM sample preparation. The advantages of FIB over more conventional methods include: high throughput, high precision, less preferential thinning and large electron transparent areas with near parallel sides. However, while providing these benefits, FIB in general produces TEM samples which are less suitable for high performance analytical (S)TEM (HRTEM, HRSTEM, high spatial resolution EELS and EDX). Relatively high energy and heavy Ga ions cause significant ion beam damage limiting the resultant TEM sample quality [2].

There are two main techniques for FIB TEM sample preparation [3]: 'pre-thin' and 'lift-out'. For the pre-thin method, a sample is first mechanically ground/polished or cut with a dicing saw and then mounted on a half grid, placed in a FIB and thinned down to electron transparency. For the liftout, the TEM lamella is cut in the FIB directly from the whole object (as large as $300 \mathrm{~mm}$ semiconductor wafer), then the object is taken out of the FIB and the TEM lamella is plucked out with a micromanipulator under an optical microscope and then transferred to a carbon coated copper grid. Both techniques have their advantages and disadvantages. Lift-out technique is less destructive but on average about $25 \%$ of the samples are lost during the plucking stage and also the carbon support film often obstructs the (S)TEM observation and hampers EELS and EDX analysis. In addition to this, post-FIB cleaning and refibbing are not possible. Pre-thin technique is more destructive, involves the mechanical treatment but it allows for a subsequent refibbing or a post-FIB cleaning of the sample (in a plasma cleaner or in a broad beam ion mill).

Recently developed in-situ plucking techniques effectively combine the benefits of both the pre-thin and the lift-out techniques allowing for all steps of sample preparation (suitable for general (S)TEM) to be carried out inside the FIB chamber in a vacuum environment. After leaving the chamber, the sample is ready to be mounted in the TEM holder. If initial TEM inspection shows that the sample needs additional thinning it can be always placed back in the FIB or transferred to a broad beam ion mill and thinned or polished to the desired thickness. In addition to that, the resulting samples can be readily cleaned in a plasma cleaner to remove the surface hydrocarbons which is required by applications that use a focused beam of high intensity (STEM, EELS, EDX).

We are currently evaluating a combination of a FIB system (FEI Strata 235 Dual Beam) with an in-situ plucking micromanipulator (Omniprobe GPP 100) to produce TEM samples which can be further polished in a low-angle low-voltage broad ion beam system (Gentle Mill from Technoorg-Linda). The set of images in Fig. 1(a-f) illustrates the main steps of in-situ plucking and resulting TEM images of a sample before and after post-FIB broad beam ion polishing are shown in Fig. 2(a-d). Fig. 3(a-c) shows the importance of plasmacleaning for focused-beam applications.

References

[1] M.H.F. Overwijk, F.C. van den Heuvel, and C.W.T. Bulle-Lieuwma, J. Sci. Technol.,B 11(6) (1993)2021

[2] J. Mardinly, Microsc. Microanal. 7 (Suppl 2: Proceedings), (2001)510

[3] L.A. Giannuzzi, F.A. Stevie, Micron, 30 (1999)197 

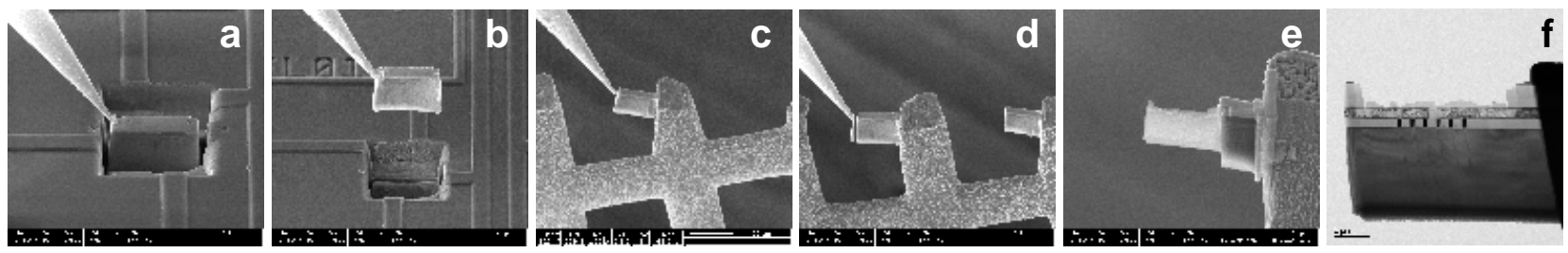

FIG. 1 (a-e) Main steps of in-situ plucking and fibbing. Several samples can be mounted and thinned on one grid (d). (f) Low magnification TEM of prepared sample.
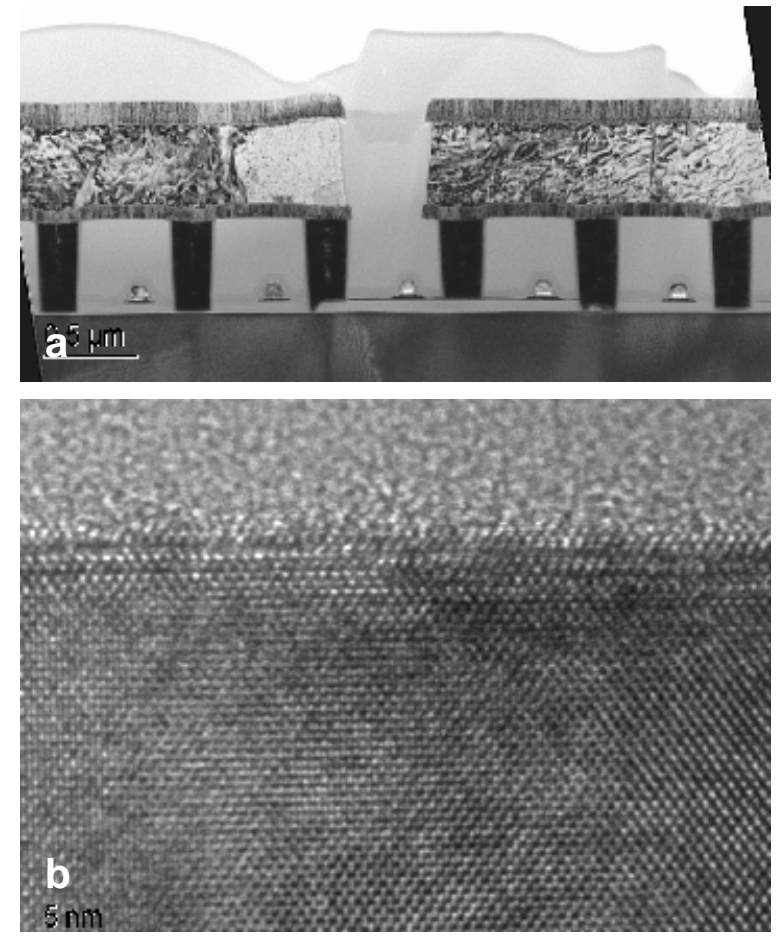
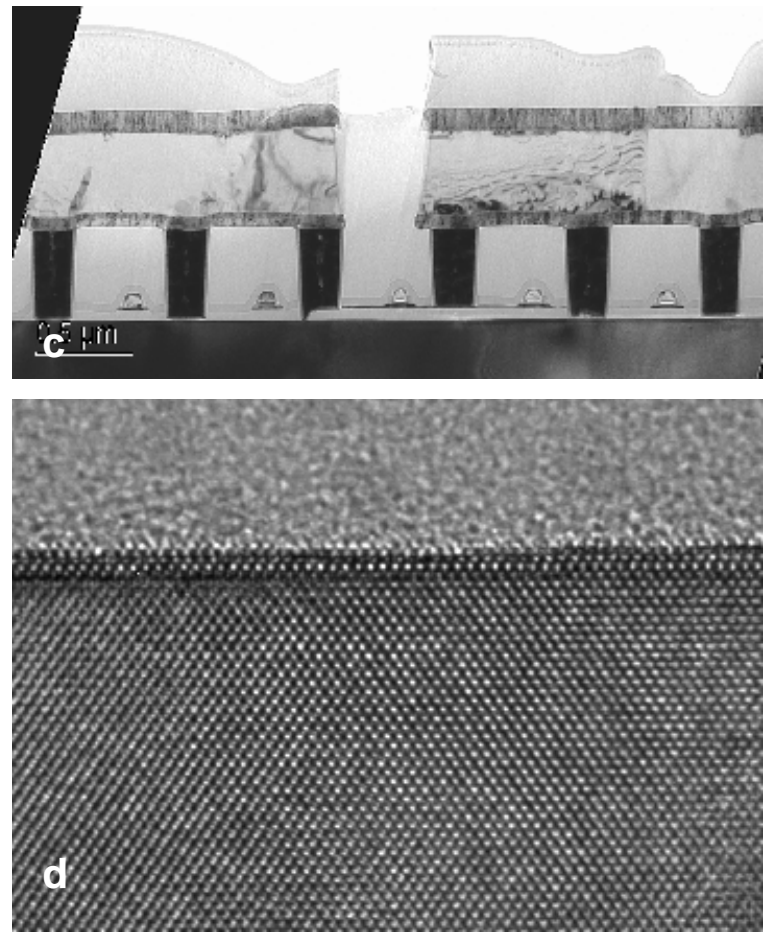

FIG. 2 (a,b) TEM and HRTEM of fibbed sample. (c,d) TEM and HRTEM of the same sample cleaned in low-angle low-energy ion mill showing a significant improvement in the sample/image quality.
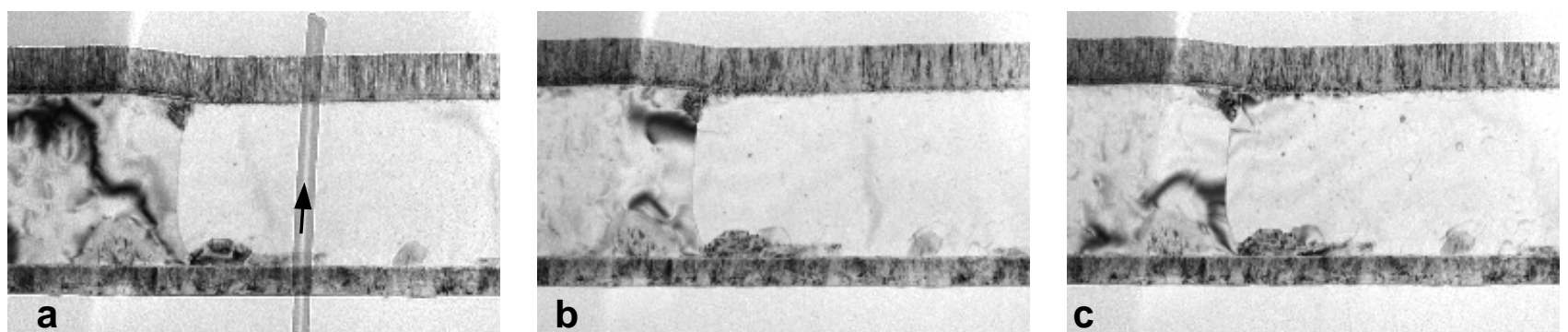

Fig. 3 (a) TEM of the fibbed sample after a STEM linescan showing a trace of carbon contamination (arrow) left by the focused electron beam; (b) same area after plasma cleaning showing that the contamination trace is removed; (c) same area after plasma cleaning and another STEM scan with the same beam current and dwell time as in (a). No trace of contamination is left proving that the sample is perfectly clean. 